\title{
Protocolo de atendimento odontológico em pacientes com múltiplas desordens sistêmicas: revisão de literatura
}

\author{
Dental care protocol in patients with multiple systemic disorders: literature review
}

Protocolo de atención odontológica en pacientes con múltiples trastornos sistémicos: revisión de la literatura

Juliana Santana Andrade ${ }^{1 *}$, Keilyane Pimenta de Melo ${ }^{1}$, lara Natividade Pereira1 ${ }^{1}$, Lívia Coutinho Varejão².

\section{RESUMO}

Objetivo: Demonstrar a importância de um atendimento odontológico específico através de um protocolo adequado para pacientes com necessidades especiais: Diabéticos, Hipertensos e Cardiopatas. Revisão bibliográfica: As inúmeras alterações fisiológicas de pacientes com múltiplas desordens sistêmicas têm grande repercussão na cavidade oral. A Diabetes mellitus (DM) é uma doença crônica causada pela falha na produção de insulina, ocasionando o descontrole na quantidade de glicose no sangue, causa xerostomia, aumento do risco de periodontite, reparo tecidual alterado e infecções fúngicas como a candidíase oral. Normalmente a DM está associada à Hipertensão Arterial Sistêmica (HAS), que é caracterizada por elevados níveis da pressão arterial, é uma doença crônica, multifatorial e silenciosa, afeta cerca de um terço da população e juntas consistem nos principais fatores de risco para as doenças Cardíacas. As alterações cardiovasculares necessitam de um gerenciamento odontológico seguro, podendo ocorrer angina e arritmias cardíacas antes ou durante o tratamento bucal e a endocardite infecciosa no pós-tratamento. Considerações finais: É indispensável o conhecimento do cirurgião dentista sobre o tratamento odontológico adequado nos pacientes com múltiplas desordens sistêmicas, a fim de prevenir os potenciais riscos de complicações e emergências resultantes de um atendimento inapropriado.

Palavras-chave: Diabetes mellitus, Hipertensão, Cardiopatias.

\section{ABSTRACT}

Objective: Demonstrate the importance of specific dental care through an appropriate protocol for patients with special needs: Diabetics, Hypertensive and Cardiac patients. Bibliographic Review: The numerous physiological changes in patients with multiple systemic disorders have great repercussions in the oral cavity. Diabetes mellitus (DM) is a chronic disease caused by the failure in insulin production, causing uncontrolled amounts of glucose in the blood, causing xerostomia, increased risk of periodontitis, altered tissue repair and fungal infections such as oral candidiasis. Usually DM is associated with Systemic Arterial Hypertension (SAH), which is characterized by high levels of blood pressure, is a chronic, multifactorial and silent disease, affects about a third of the population and together are the main risk factors for heart diseases. Cardiovascular changes require safe dental management, and angina and cardiac arrhythmias may occur before or during oral treatment and post-treatment infective endocarditis. Final considerations: The dentist's knowledge of the proper dental treatment in patients with multiple systemic disorders is essential in order to prevent the potential risks of complications and emergencies resulting from inappropriate care.

Keywords: Diabetes mellitus, Hypertension, Heart disease.

\section{RESUMEN}

Objetivo: Demostrar la importancia de la atención odontológica específica mediante un protocolo adecuado para pacientes con necesidades especiales: Diabéticos, Hipertensos y Cardiacos. Revisión bibliográfica: Los numerosos cambios fisiológicos en pacientes con múltiples trastornos sistémicos tienen gran repercusión en la cavidad bucal. La diabetes mellitus (DM) es una enfermedad crónica provocada por la insuficiencia en la producción

${ }^{1}$ Centro Universitário do Norte (UNINORTE), Manaus - AM. *E-mail: jusantanaandrade@gmail.com

${ }^{2}$ Universidade Federal do Amazonas (UFAM), Manaus - AM. 
de insulina, que provoca cantidades incontroladas de glucosa en la sangre, provoca xerostomía, aumento del riesgo de periodontitis, reparación tisular alterada e infecciones fúngicas como candidiasis oral. Habitualmente la DM se asocia con la Hipertensión Arterial Sistémica (HSA), la cual se caracteriza por niveles altos de presión arterial, es una enfermedad crónica, multifactorial y silenciosa, afecta aproximadamente a un tercio de la población y en conjunto son los principales factores de riesgo de enfermedades Cardíaco. Los cambios cardiovasculares requieren un tratamiento dental seguro y pueden producirse angina y arritmias cardíacas antes o durante el tratamiento oral y endocarditis infecciosa postratamiento. Consideraciones finales: El conocimiento del cirujano dentista sobre el tratamiento dental adecuado en pacientes con múltiples trastornos sistémicos es fundamental para prevenir los posibles riesgos de complicaciones y emergencias derivadas de una atención inadecuada.

Palabras clave: Diabetes mellitus, Hipertensión, Enfermedades del corazón.

\section{INTRODUÇÃO}

A diversidade de pacientes que buscam atendimentos odontológicos e possuem restrições médicas vem aumentando a cada ano. E com isso, muitas emergências ocorrem dentro dos consultórios por conta da falta de um preparo criterioso do cirurgião dentista, de uma anamnese detalhada e de um exame físico preciso. Uma emergência caracteriza-se pelo agravamento da saúde, ocorrendo sofrimento ao paciente e causando risco da perda de sua vida. Ocorre de forma imprevisível e requer uma ação rápida e correta. Podendo o profissional ser enquadrado na esfera civil e criminal por seus atos se constituírem imprudência, imperícia ou negligência e ser condenado por homicídio doloso e lesões corporais culposas quando o mesmo não estiver apto para solucionar as possíveis complicações decorrentes de um atendimento inapropriado (POLIZELI AF, et al., 2017; FABRIS V, et al., 2018).

Os indivíduos que apresentam quaisquer alterações físicas, emocionais ou sociais são considerados pacientes especiais. A conduta e manejo dos pacientes com alterações crônicas requer uma análise detalhada e um atendimento individualizado com o conhecimento do profissional sobre as técnicas adequadas, por isso é importante está sempre atualizado e apto para o atendimento desses pacientes (LEITE RB, et al., 2020; LÚCIO PS e BARRETO RS, 2012).

A Diabetes Melittus (DM) se caracteriza por ser uma doença crônica e sistêmica e ocorre devido aos níveis aumentados de glicose no sangue (hiperglicemia), acarretando influência em todo o organismo, inclusive na cavidade oral. Suas manifestações gerais mais comuns são observadas com o descontrole glicêmico, a perda de peso, a poliúria, a polidipsia e a polifagia. Já na cavidade oral suas alterações são facilmente identificadas como aumento do índice de doença periodontal, xerostomia, candidíase oral e viscosidade lingual. Classificase como DM tipo 1, DM tipo 2 e a Diabetes Gestacional, podendo apresentar-se compensada e descompensada e o profissional deve estar apto para identificar e analisar os riscos e benefícios da conduta para o atendimento (LABOLITA KA, et al., 2020; OLIVEIRA MF, et al., 2019; SILVA GR, et al., 2019; COSTA RM, et al., 2016).

A Hipertensão Arterial Sistêmica (HAS) é uma condição muito comum entre a população, caracterizada pela elevação anormal dos níveis da pressão arterial, podendo ser sistólica e diastólica. Trata-se de uma doença silenciosa, e por este motivo não apresenta grandes alterações e sintomas, o que a torna uma doença muito perigosa, pois só a sua impercepção pode ser mortal. É classificada em hipertensão primária ou essencial e secundária (SOUTHERLAND JH, et al., 2016; CALISTRO LC, et al., 2019).

Grande parte da população possui doenças cardiovasculares relacionadas a hipertensão arterial sistêmica e ao diabetes. O paciente que apresenta alterações cardíacas como arritmias, angina e endocardite infecciosa requer um protocolo específico, um manejo clínico cuidadoso, uma comunicação eficaz com o cardiologista que o acompanha e sempre está sob conduta de paciente de risco (MISTURINI L, et al., 2016; MELO LA e LIMA KC, 2020; AMARAL COF, et al., 2016; LEITE RB, et al., 2020).

Tendo em vista a alta complexidade de atendimento em pacientes com múltiplas desordens sistêmicas (diabéticos, hipertensos e cardiopatas), o objetivo deste trabalho é avaliar a literatura existente, com a finalidade de verificar a assistência odontológica e apresentar uma conduta simplificada e objetiva, que informe ao cirurgião dentista um protocolo de atendimento atualizado para esses pacientes com necessidades especiais. 


\section{REVISÃO BIBLIOGRÁFICA}

\section{Diabetes Melittus (DM)}

A Diabete Mellitus (DM) apresenta-se de três tipos: A Diabetes Mellitus tipo 1 (DM1) que se caracteriza pela destruição de células beta dentro do pâncreas, causando a deficiência da produção de insulina, se manifesta geralmente em indivíduos com menos de 25 anos e denomina-se insulino dependente. A Diabetes Mellitus tipo 2 (DM2) é a mais comum entre a população, está associada a incapacidade das células receptoras de captar a insulina expelida pelo pâncreas, ou a diminuição dessa produção e excreção. Apresenta-se como não-dependente da insulina, e geralmente seus sintomas iniciam-se após os 30 anos e suas causas são o sedentarismo, obesidade, idade, dentre outros. Já o Diabetes Mellitus Gestacional (DMG) ocorre durante a gravidez e desenvolve uma diminuição ainda maior da sensibilidade periférica à insulina e a sua produção e excreção comparada a DM2, podendo permanecer ou não após o parto (OLIVEIRA TF, et al.,2016; SILVA RG, et al., 2019; LABOLITA KA, et al., 2020; MACÊDO A, et al., 2018).

Pacientes com níveis glicêmicos de $70 \mathrm{mg} / \mathrm{dl}$ até $100 \mathrm{mg} / \mathrm{dl}$ estão dentro da normalidade, com níveis de $100 \mathrm{mg} / \mathrm{dl}$ até $125 \mathrm{mg} / \mathrm{dl}$ apresentam um alto risco para DM e deve-se repetir o teste para confirmação. Referente aos riscos, os níveis glicêmicos de pacientes diabéticos descompensados classificados como ASA III para procedimentos são: Glicemia menor que $150 \mathrm{mg} / \mathrm{dl}$ é considerado pequeno risco, em procedimentos não-cirúrgicos mantem-se as precauções rotineiras. Em procedimentos cirúrgicos adiciona adequação da dosagem de insulina e sedação auxiliar (COSTA RM, et al., 2016; RIBEIRO DAS e SOUZA AO, 2019; SILVA RG, et al., 2019; OLIVEIRA MF, et al., 2019).

Glicemia menor que $250 \mathrm{mg} / \mathrm{dl}$ é considerado médio risco e em procedimentos não-cirúrgicos utilizar a sedação auxiliar e para as cirurgias de pequeno porte o ajuste da insulina se faz necessário e a possibilidade de internação para amenizar possíveis complicações. Glicemia maior que $250 \mathrm{mg} / \mathrm{dl}$ se enquadra em procedimentos de alto risco e o tratamento deve ser adiado até o completo equilíbrio de suas condições metabólicas, exceto em tratamento de urgência ou paliativo. O exame de Hemoglobina Glicada é indicado para avaliar os níveis glicêmicos dos pacientes diabéticos (SILVA RG, et al., 2019; OLIVEIRA MF, et al., 2019).

É possível identificar os sinais e sintomas da DM durante a consulta com o cirurgião dentista. $\mathrm{Na}$ Anamnese, que é a primeira avaliação do paciente, detalha-se as possíveis alterações sentidas por ele como: sede excessiva (polidipsia), aumento da frequência e volume urinário (poliúria), aumento da fome (polifagia) e o relato da perda de peso. Com o exame físico na cavidade oral, também é possível a identificação dos seguintes sintomas: doenças periodontais que resultam em perda de inserção dental, reabsorção óssea e sangramento durante a sondagem, xerostomia (sensação de boca seca), hálito cetônico, infecções fúngicas como a candidíase oral e a dificuldade de cicatrização (OLIVEIRA TF, et al.,2016; LABOLITA KA, et al., 2020).

$O$ atendimento odontológico deve iniciar pelas orientações de higiene bucal. As consultas e procedimentos devem ser preferencialmente pela manhã, pois é quando ocorre a máxima secreção da insulina. O paciente deve comparecer ao consultório alimentado. Deve-se aferir a pressão arterial antes e depois do tratamento ou consulta. Os tratamentos devem ser de curta duração, diminuindo os quadros hipoglicemia, porém, se não for possível, o cirurgião dentista deve pausar 0 atendimento e oferecer uma pequena quantidade de carboidrato a fim de reestabelecer seu nível glicêmico. A hipoglicemia pode-se apresentar com sintomas de fraqueza, palpitações, sudorese, tremores, alterações de consciência entre outros. O medo e a ansiedade do paciente devem ser evitados pois causam a elevação da glicemia (hiperglicemia) através da liberação de adrenalina (OLIVEIRA TF, et al., 2016; LABOLITA KA, et al., 2020; COSTA RM, et al., 2016; MACÊDO A, et al., 2018).

Os pacientes diabéticos submetidos a procedimentos que induzam a possibilidade de dor são indicados a prescrição de analgésico: Dipirona ou paracetamol. Em casos mais graves, as preparações com codeína são indicadas. Os antiinflamatórios de escolha são Betametasona ou Dexametasona em dose única de $4 \mathrm{mg}$. Os antibióticos não possuem contraindicações importantes para os diabéticos, salvo as do próprio fármaco e os mais indicados são as penicilinas ou cefalosporinas, e deve ser requisitado após procedimento invasivo para diabéticos descompensados devido ao risco elevado de infecções e inflamações, auxiliando no reparo tecidual (LABOLITA KA, et al., 2020; SILVA RG, et al., 2019). 
Em casos em que haja necessidade de profilaxia antibiótica, postula-se a dose única de Amoxicilina 1G ou Clindamicina $600 \mathrm{mg}$ em caso de alérgicos a penicilina, 1 hora antes do procedimento. $O$ anestésico mais indicado é a Prilocaína 3\% com Felipressina, porém seu uso é contraindicado para gestantes, e em casos de pacientes anêmicos avalia-se os riscos de metemoglobinemia, a Articaína 4\% com epinefrina 1:100000 também apresenta indicação. Os enxaguantes bucais ou colutórios são indicados para pacientes que apresentam xerostomia, mas não devem possuir álcool em sua composição, aumentando o risco de lesões orais (SHUEROFF EF, et al., 2016; RIBEIRO DAS e SOUZA AO, 2019).

O digluconato de clorexidina, o cloreto de cetilpiridino, o tricosan e os óleos essenciais são alguns que não possuem álcool na sua composição. Em casos de pacientes muito ansiosos, um ansiolítico pode ser administrado 45 min antes. Não são indicados a utilização do ácido acetilsalicílico (AAS) e antiinflamatórios não-asteroidais (AINE'S), exceto após a troca de informações com o médico do paciente (LABOLITA KA, et al., 2020; SILVA RG, et al., 2019; OLIVEIRA TF, et al.,2016; SHUEROFF EF, et al., 2016; RIBEIRO DAS e SOUZA AO, 2019; FABRIS V, et al., 2018).

Conforme afirma Labolita KA et al., (2020), é de suma importância o cirurgião-dentista ter o conhecimento as complicações durante o tratamento de Diabetes Millitus devido à alta incidência nos consultórios, adotando condutas clínicas adequadas e individualizadas conhecendo os aspectos clínicos, etiológicos e patogênicos da doença, promovendo assim, o bem-estar e melhores condições de saúde desse paciente. De acordo com Priyanka et al., (2019) nas consultas realizadas durante as manhãs, observou-se que a taxa média de pulso (PR) e a média de sangue sistólico e diastólico da pressão (PAS) são maiores no início da manhã em todos os grupos durante atendimento.

No estudo de Délgado-Pérez et al., (2017) relatou uma população de adultos mexicanos que foram diagnosticados com DM2 ou hipertensão relacionando essas doenças com perca de elementos dentais os quais vivendo em seus afazeres cotidiano de forma independente. Foi constatado que apesar de controlar vários fatores as pessoas tiveram número médio maior de dentes perdidos entre eles em primeiro lugar o trauma e a condição sistêmica em que DM2 e a hipertensão associadas causam a saúde bucal, segundo a falta de orientações adequadas a esses pacientes, levando-os a tratamentos odontológicos não conservadores e extrações dentárias aliadas aos tratamentos (iatrogenia). Achados de Oliveira et al., (2018) apontam que as políticas públicas estão cada vez mais direcionadas a atenção básica para evitar e atingir a cultura da naturalização da perda dentária/protesismo que traz danos a qualidade de vida de pacientes hipertensos e diabéticos no que diz respeito a alimentação, mastigação e saúde bucal.

\section{Hipertensão Arterial Sistêmica (HAS)}

A hipertensão ocorre devido a descompensação da pressão arterial (PA), é silenciosa, crônica e pode ser fatal se não tratada. Apresenta-se como A Hipertensão Arterial Estágio I, quando os valores se aproximam de 140-159 da pressão sistólica e próximos aos 90-99 mmHg na diastólica. A Hipertensão Arterial Estágio II se os valores estão em 160-171 e 100-109 mmHg respectivamente. E a Hipertensão estágio III com valores maiores que 180/110 mmHg. Alguns pacientes normosistêmicos apresentam um elevado nível de PA dentro do consultório, caracterizando-se como hipertensão do avental branco, com resultados de monitoramentos em casa de $135 / 85 \mathrm{mmHg}$ ou no ambulatório de $130 / 80 \mathrm{mmHg}$ após $24 \mathrm{~h}$ do atendimento (SHUEROFF EF, et al., 2016; SOUTHERLAND JH, et al., 2016).

O grande problema de muitos consultórios é que a aferição da PA prévia as consultas não é uma rotina devido ao grande fluxo de pacientes, mesmo a maioria dos pacientes hipertensos sendo assintomáticos e sendo conhecido como "assassino silencioso". Isso traz um risco elevado de complicações na cadeira odontológica haja vista que esses pacientes necessitam de um protocolo de atendimento diferenciado (FABRIS V, ET AL., 2018; PRIYANKA, et al., 2019; BOGARI DF, et al., 2016).

As manifestações orais em pacientes HAS são frequentes devido ao uso de anti-hipertensivos e podem apresentar-se como: estomatites, glossite, paladar alterado, inflamação das glândulas salivares e hiperplasia gengival (inflamação da língua), dor na ATM, língua escurecida, angioedema da língua, mucosa, lábios e face. As orientações quanto as suas condições clínicas e possíveis complicações são fundamentais. As manifestações mais comuns são: alteração no fluxo salivar, candidíase oral e lesões de cárie. 
Em caso de crise hipertensiva durante 0 atendimento odontológico onde os valores ultrapassem a $180 \mathrm{mmHg} / 120 \mathrm{mmHg}$ o protocolo a ser seguido é: interrupção imediata do tratamento devido ao alto risco de aumento de sangramento, , coloque o paciente sentado confortavelmente, monitorar os sinais vitais, administrar captopril de 25 a $50 \mathrm{mg}$ por via sublingual se necessário, quando amenizar a crise o paciente deve ser encaminhado para o hospital imediatamente como urgência hipertensiva (CALISTRO LC, et al., 2019; SOUTHERLAND JH, et al., 2016; OLIVEIRA EJ, et al., 2018).

É de suma importância que o paciente seja hipertenso controlado, pois as complicações dos procedimentos são minimizadas, podendo ser utilizado, com cautela, o mesmo protocolo de atendimento de pacientes normossistêmicos. O limite de PA para atendimento é de $140 / 90 \mathrm{mmHg}$, acima desses valores o atendimento deve ser evitado até a normalização da PA. Para os hipertensos controlados o anestésico com vasoconstritor Prilocaína 3\% com Felipressina pode ser utilizado, limitando-se a 3 tubetes (NASCIMENTO EM et al., 2011).

Em caso de urgência de paciente descompensado o mais indicado é a Mepivacaína $3 \%$ sem vasoconstritor. Se o paciente não estiver medicado e em caso de pacientes descompensados com pressão arterial acima de 140/95 mmHg não se deve intervir, recomenda-se que busque assistência terapêutica em pronto atendimento para evitar intercorrências. Os benzodiazepínicos e técnicas de relaxamento ou condicionamento psicológico são utilizados quando o paciente se encontra muito apreensivo com 0 tratamento, evitando a alteração de seus níveis de PA (SILVA CH, et al., 2019).

Os antiinflamatórios AINES não são indicados pois provoca a retenção de sódio e consequentemente a elevação da pressão arterial em decorrência do aumento do volume de liquido intravascular. Analgésicos que contenham AAS são contra-indicados pois interagem com anti-hipertensivos a exemplo do Captopril (NASCIMENTO EM et al., 2011; SILVA CH, et al., 2019).

Como descrito por Calistro LC et al., (2019), o tratamento odontológico de pacientes hipertensos representa um desafio para os cirurgiões-dentistas, que devem estar fundamentados cientificamente para estabelecer as melhores condutas para o manejo desse tipo de paciente. Realizar a anamnese e aferição da pressão arterial, orientar o paciente sobre sua condição sistêmica, esclarecer como terapias empregadas e reconhecer como mudanças resultantes delas são fundamentais para estabelecer o melhor plano de tratamento e minimizar ou prevenir problemas que possam ocorrer durante $\mathrm{o}$ atendimento odontológico.

Para Rodrigues KP et al., (2015) as alterações clinicas dos pacientes de HAS e o DM deve ser percebidas pelo cirurgião-dentista pois são doenças com uma alta taxa de prevalência entre a população e Southerland $\mathrm{JH}$ et al, (2016) reforça essa afirmação, destacando que o profissional deve estar sempre atualizado sobre qual a melhor conduta para manejos desses pacientes, pois novas informações são publicadas frequentemente. Os descritos de Oliveira TF et al., (2016) robustecem esses apontamentos de Labolita et al, (2020) salientando que durante o atendimento odontológico, uma criteriosa anamnese é indispensável para a decisão das condutas terapêuticas e realização correta dos procedimentos. Recomenda-se especial atenção aos tipos de medicamentos utilizados pelo paciente, bem como à escolha dos anestésicos locais.

\section{Cardiopatas}

Uma das principais causas de morte são as doenças cardíacas. As complicações decorrentes de um tratamento odontológico em pacientes cardiopatas podem ser irreversíveis. Dentre muito problemas que podem ocorrer antes, durante e após o atendimento odontológico, as manifestações clínicas da arritmia cardíaca, a angina e a endocardite infecciosa devem ser identificadas e tratadas corretamente (LEITE RB, et al., 2020).

A arritmia cardíaca é a alteração dos batimentos cardíacos do paciente. A grande maioria das arritmias cardíacas não representam preocupações para o paciente e cirurgião dentista, porém existem arritmias que podem resultar em risco elevado como o infarto do miocárdio, angina instável e morte súbita. O controle da ansiedade deve ser efetivo e a monitoração do pulso do paciente deve ser constante. A angina é característica de uma sensação de aperto doloroso ou pressão, na região do osso esterno (parte anterior do tórax) e irradia para o pescoço, braço esquerdo, mandíbula e língua. Ela é classificada com angina estável e instável (LEITE $\mathrm{RB}$, et al., 2020). 
A do tipo estável ocorre durante atividade física ou algum tipo de estresse emocional, é de curta duração, cerca de cinco minutos e o alivio ocorre quando o fator desencadeante é removido. Já a do tipo instável a dor é mais intensa, não finda com a remoção do fator desencadeante, é progressiva e em repouso pode ser precipitada. Durante um evento de angina, é muito importante saber diferenciar qual tipo está ocorrendo para lançar mão da melhor conduta. Se for a angina estável e o tratamento tiver que continuar, o paciente deve ficar em posição semi-supina, analisando sempre o nível de estresse do paciente (LEITE RB, et al., 2020; MISTURINE L, et al., 2016).

É importante entender que na ocorrência de um episódio de angina deve-se administrar por via sublingual um vasodilatador coronariano juntamente com oxigênio, o procedimento deve cessar e se a dor permanecer por mais cinco minutos deve-se administrar o segundo comprimido sublingual, se a dor não regredir por cerca de quinze minutos o paciente deve ser encaminhado para o hospital com suspeita de Infarto do Miocárdio (IM) (MISTURINE L, et al., 2016).

A endocardite infecciosa (EI) ocorre nos tecidos cardíacos e a American Heart Association em suas diretrizes propõe a profilaxia antibiótica em pacientes que se enquadram nos casos de valvas protéticas, nos que já tiveram El anteriormente, transplantados cardíacos com valvulopatia cardíaca e doença cardíaca congênita. No consultório a profilaxia antibiótica é feita 1 hora antes do procedimento com $2 \mathrm{~g}$ de Amoxicilina via oral ou a Clindamicina $600 \mathrm{mg}$ para alérgicos a penicilina, para procedimentos que ocorra perfuração da mucosa oral como a biópsia, manipulação dos tecidos gengivais e da região periapical (LEITE RB, et al., 2020; MISTURINE L, et al., 2016).

O anestésico de escolha é a prilocaína $3 \%$ com a felipressina com no máximo até 5 tubetes por sessão, pois não age sobre os receptores adrenérgicos e não alteram a pressão arterial. Alguns estudos mostram a segurança da lidocaína $2 \%$ com epinefrina com o limite máximo de 2 tubetes. Após o procedimento odontológico, obter o cuidado com a hipotensão ortostática é fundamental (LEITE RB, et al., 2020; MISTURINE L, et al., 2016; SANTOS-PAUL MA, et al., 2015). Segundo Leite RB et al., (2020) é de grande importância o cirurgião-dentista conhecer os aspectos mais relevantes para atendimento de pacientes cardiopatas no consultório odontológico, assimilando como fator de risco o diagnóstico da hipertensão arterial sistêmica, assegurando o melhor tratamento e conduta frente a esses pacientes.

\section{CONSIDERAÇÕES FINAIS}

O tratamento odontológico para pacientes Diabéticos, Hipertensos e Cardiopatas é desafiante, por isso, o cirurgião dentista deve estar embasado cientificamente para estabelecer o melhor atendimento e tratamento. A necessidade de um protocolo de atendimento correto é devido à grande demanda de pacientes com múltiplas desordens sistêmicas e tal conhecimento transmite mais segurança no manejo, pois o reconhecimento de tais distúrbios é indispensável para que executem um atendimento diferenciado, com melhores condições de cuidado e consequentemente evitando maiores complicações em decorrência do tratamento odontológico inadequado.

\section{REFERÊNCIAS}

1. AMARAL CO, et al. Oral health evaluation of cardiac patients admitted to cardiovascular presurgery intervention. RGO, Rev Gaúch Odontol, Porto Alegre, 2016, 64 (4): 419-424.

2. BOGARI DF, et al. The prevalence of hypertension in endodontic clinics: a pilot study. Dentistry, 2016, 6(4): 1-12.

3. CALISTRO, LC, et al. Dental care in hypertense patients: systematic review. Brazilian Journal of Implantology and Health Sciences, 2019, 1(6): 152-168.

4. COSTA RM, et al. O paciente diabético na clínica odontológica: Diretrizes para o acolhimento e atendimento. Revista Brasileira de Ciências da Saúde, 2016; 20(4): 333-340.

5. DELGADO-PÉREZ VJ, et al. Diabetes or Hypertension as Risk Indicators for Missing Teeth Experience: An Exploratory Study in a Sample of Mexican Adults. Nigerian Journal of Clinical Practice, 2017; 20(10): 1336-1341.

6. FABRIS V, et al. Conhecimento dos cirurgiões dentistas sobre o uso de anestésicos locais em pacientes: diabéticos, hipertensos, cardiopatas, gestantes e com hipertireoidismo. Journal of Oral Investigations, Passo Fundo, 2018, 7(1): 33-51.

7. LABOLITA KA, et al. Assistência odontológica à pacientes diabéticos. Ciências Biológicas e de Saúde Unit, Alagoas, 2020, 6(1): 89-98.

8. LEITE RB, et al. Avaliação do conhecimento de cirurgiões dentistas diante do atendimento a cardiopatas graves na atenção primária. Revista Odontológica de Araçatuba, 2020, 41(1): 09-14. 
9. LÚCIO PS, BARRETO RS. Emergências Médicas no Consultório Odontológico e a (In)Segurança dos Profissionais. R bras ci Saúde, 2012, 16(2): 267-272.

10. MACÉDO A, et al. Contribution of chronic diseases to the prevalence of disability in basic and instrumental activities of daily living in elderly Brazilians: The National Health Survey (2013). Cad. Saúde Pública, Rio de Janeiro, 2018, 34(1): 00204016.

11. MELO LA, LIMA KC. Fatores associados às multimorbidades mais frequentes em idosos brasileiros. Ciênc. saúde coletiva, Rio de Janeiro, 2020, 25(10): 3879-3888.

12. MISTURINI L, et al. Doenças cardiovasculares e sua interação com a odontologia - relato de caso clínico. Ação Odonto, 2016, 1(2): 13-16.

13. NASCIMENTO EM, etc al. Abordagem odontológica de pacientes com hipertensão - um estudo de intervenção, 2011, 16(1).

14. OLIVEIRA EJ, et al. Qualidade de vida e condições de saúde bucal de hipertensos e diabéticos em um município do Sudeste Brasileiro. Ciência \& Saúde Coletiva, 2018, 23(3): 763-772.

15. OLIVEIRA MF, et al. Cuidados odontológicos em pacientes diabéticos. Arq. Catarin Med, 2019, 48(3): $158-170$.

16. OLIVEIRA TF, et al. Conduta odontológica em pacientes diabéticos: considerações clínicas. Odontol. Clín.-Cient, Recife, 2016, 15(1): 13 - 17.

17. POLIZELI AF. et al. Emergências médicas em consultório odontológico: implicações éticas e legais para o cirurgiãodentista. Journal of Multidisciplinary Dentistry, 2020, 10(1): 59-64.

18. PRIYANKA E. et al. Evaluation of changes in Blood pressure and Pulse rate of hypertensive patient during early morning and evening dental appointments. Journal of Advanced Medical and Dental Sciences Research, 2019, 7(2).

19. RIBEIRO DA, SOUZA AO. Cuidados odontológicos em pacientes idosos portadores de diabetes mellitus. Trabalho de Conclusão de Curso (Bacharel em Odontologia) - Centro Universitário do Planalto Central Apparecido dos Santos, 2019.

20. RODRIGUES KP. et al. Percepção de acadêmicos de Odontologia sobre seus conhecimentos para o atendimento odontológico de hipertensos e diabéticos. Revista da ABENO, 2015, 15(4), 19-28.

21. SANTOS-PAUL MA, et al. Local anesthesia with epinephrine is safe and effective for oral surgery in patients with type 2 diabetes mellitus and coronary disease: a prospective randomized study. Clinics, São Paulo, 2015, 70(3), 185-189.

22. SCHUEROFF EF, et al. Importância do conhecimento do cirurgião dentista sobre pressão arterial, fatores modificadores e complicações sistêmicas durante atendimento cirúrgico. Arquivos do MUDI, 2016, 20 (3): 44-58.

23. SILVA CH, et al. Atendimento odontológico a hipertensos e diabéticos na atenção primária à saúde. Destaques Acadêmicos, Lajeado, 2019, 11(3): 152-164.

24. SILVA RG, et al. Atendimento odontológico ao paciente diabético. Rev. UNINGÁ, Maringá, 2019, 56(S3): $158-168$.

25. SOUTHERLAND JH, et al. Dental management in patients with hypertension: challenges and solutions. Clinical, Cosmetic and Investigational Dentistry, 2016, 8(1): 111-120. 